\title{
EQUIPARTITION OF ENERGY FOR A CLASS OF SECOND ORDER EQUATIONS
}

\author{
DAVID G. COSTA
}

\begin{abstract}
We consider the Cauchy problem for a class of second order equations of the form $\left(d / d t-A_{2}\right)\left(d / d t-A_{1}\right) u(t)=0$ in a Hilbert space $H$. A d'Alembert type solution formula is presented and we give a suitable definition of energy. Also, we derive a necessary and sufficient condition for the asymptotic equipartition of energy (Kinetic and Potential) to hold. These results generalize corresponding results for the abstract wave equation $\left(d^{2} / d t^{2}+A^{2}\right) u(t)=0$.
\end{abstract}

1. Introduction. If $a_{1} \neq a_{2}$ are complex numbers, the general solution of the ordinary differential equation

$$
u^{\prime \prime}-\left(a_{1}+a_{2}\right) u^{\prime}+a_{1} a_{2} u=0
$$

is given by

$$
\begin{aligned}
u(t)= & \exp \left(t a_{1}\right) c_{1}+\exp \left(t a_{2}\right) c_{2} \\
= & \frac{1}{2}\left[\exp \left(t a_{1}\right)+\exp \left(t a_{2}\right)\right]\left(c_{1}+c_{2}\right) \\
& +\frac{1}{2}\left[\exp \left(t a_{1}\right)-\exp \left(t a_{2}\right)\right]\left(c_{1}-c_{2}\right),
\end{aligned}
$$

where $c_{1}, c_{2}$ are arbitrary constants. In addition, if initial data $u(0)=u_{0}, u^{\prime}(0)$ $=u_{1}$ are prescribed, then $c_{1}$ and $c_{2}$ can be determined from the two equations $c_{1}+c_{2}=u_{0}, a_{1} c_{1}+a_{2} c_{2}=u_{1}$ and, hence, the unique solution of (*) in this case is given by the formula

$$
\begin{aligned}
u(t)= & \frac{1}{2}\left[\exp \left(t a_{1}\right)+\exp \left(t a_{2}\right)\right] u_{0} \\
& +\left[\exp \left(t a_{1}\right)-\exp \left(t a_{2}\right)\right]\left(a_{1}-a_{2}\right)^{-1}\left(u_{1}-\frac{a_{1}+a_{2}}{2} u_{0}\right) .
\end{aligned}
$$

Notice that, in view of the identity

$$
\exp \left(t a_{1}\right)-\exp \left(t a_{2}\right)=\int_{0}^{t} \exp \left((t-s) a_{1}\right) \exp \left(s a_{2}\right)\left(a_{1}-a_{2}\right) d s
$$

we can rewrite (1) as

$$
\begin{aligned}
u(t)= & \frac{1}{2}\left[\exp \left(t a_{1}\right)+\exp \left(t a_{2}\right)\right] u_{0} \\
& +\int_{0}^{t} \exp \left((t-s) a_{1}\right) \exp \left(s a_{2}\right)\left(u_{1}-\frac{a_{1}+a_{2}}{2} u_{0}\right) d s,
\end{aligned}
$$

Received by the editors May 22, 1974.

AMS (MOS) subject classifications (1970). Primary 34G05; Secondary 47B25. 
this latter expression having the advantage that it is meaningful even if $a_{1}=a_{2}$.

This d'Alembert type formula suggests a similar one for the Cauchy problem associated with the abstract equation

$$
\left(d / d t-A_{2}\right)\left(d / d t-A_{1}\right) u(t)=0
$$

in a Hilbert space $H$. We shall do this in the next section, where we also give a definition of "energy" and show it is preserved for all times. In §3, a necessary and sufficient condition in order to have equipartition of energy is given. Similar results for the wave equation $\left(A_{1}=-A_{2}=i A, A\right.$ selfadjoint $)$ can be found in [1], [2], [5].

2. Representation of a solution and energy. Let $A_{1}, A_{2}$ be skewadjoint operators on a Hilbert space $H$ (with scalar product $(\cdot, \cdot)$ and norm $\|\cdot\|$ ) and $\left\{T_{1}(t) \mid t \in \mathbf{R}\right\},\left\{T_{2}(t) \mid t \in \mathbf{R}\right\}$ the unitary groups they generate, respectively. We assume that

$$
T_{1}(t) T_{2}(s)=T_{2}(s) T_{1}(t)
$$

for all $t, s \in \mathbf{R}$. Then it is easy to see that an analogue of (2) holds, namely,

(6) $\left[T_{1}(t)-T_{2}(t)\right] v=\int_{0}^{t} T_{1}(t-s) T_{2}(s)\left(A_{1}-A_{2}\right) v d s \quad \forall v \in \mathscr{D}\left(A_{1}\right) \cap \mathscr{D}\left(A_{2}\right)$.

From now on, we shall assume that $\mathscr{D}_{\infty}=\cap_{k=1}^{\infty} \mathscr{D}_{k}$ is dense in $H$, where $\mathscr{D}_{k}=\bigcap_{j_{i} \in\{1,2\}} \mathscr{D}\left(A_{j_{1}} \cdots A_{j_{k}}\right), k=1,2, \ldots$, and we make the following

Definition. A function $u: \mathbf{R} \rightarrow H$ is a solution of (4) if $u \in C^{1}(\mathbf{R}, H)$ $\cap C^{0}\left(\mathbf{R}, \mathscr{D}\left(A_{1}\right)\right)$ and $v_{1}=d u / d t-A_{1} u \in C^{1}(\mathbf{R}, H) \cap C^{0}\left(\mathbf{R}, \mathscr{D}\left(A_{2}\right)\right)$ with $d v_{1} / d t-A_{2} v_{1}(t)=0 \forall t \in \mathbf{R}$.

Then the Cauchy problem for (4) with initial data $u(0)=u_{0} \in \mathscr{D}_{2}, u^{\prime}(0)$ $=u_{1} \in \mathscr{D}_{1}$ has its solution (it is clearly unique) given by the formula

$$
u(t)=\frac{1}{2}\left[T_{1}(t)+T_{2}(t)\right] u_{0}+\int_{0}^{t} T_{1}(t-s) T_{2}(s)\left(u_{1}-\frac{A_{1}+A_{2}}{2} u_{0}\right) d s
$$

Indeed, since $u_{0} \in \mathscr{D}_{2}, u_{1} \in \mathscr{D}_{1}$ and in view of (5), we have that

$$
u \in C^{1}(\mathbf{R}, H)
$$

with

$$
\begin{aligned}
u^{\prime}(t)= & \frac{1}{2}\left[T_{1}(t) A_{1}+T_{2}(t) A_{2}\right] u_{0} \\
& +\int_{0}^{t} T_{1}(t-s) T_{2}(s) A_{1}\left(u_{1}-\frac{A_{1}+A_{2}}{2} u_{0}\right) d s \\
& +T_{2}(t)\left(u_{1}-\frac{A_{1}+A_{2}}{2} u_{0}\right) .
\end{aligned}
$$

Also, $u \in C^{0}\left(\mathbf{R}, \mathscr{D}\left(A_{1}\right)\right)$, with 


$$
\begin{aligned}
A_{1} u(t)= & \frac{1}{2}\left[T_{1}(t) A_{1}+T_{2}(t) A_{1}\right] u_{0} \\
& +\int_{0}^{t} T_{1}(t-s) T_{2}(s) A_{1}\left(u_{1}-\frac{A_{1}+A_{2}}{2} u_{0}\right) d s,
\end{aligned}
$$

and hence, $v_{1}(t)=u^{\prime}(t)-A_{1} u(t)=T_{2}(t)\left(u_{1}-A_{1} u_{0}\right)$. Again, our hypothesis yields $v_{1} \in C^{1}(\mathbf{R}, H)$ with $v_{1}^{\prime}(t)=T_{2}(t) A_{2}\left(u_{1}-A_{1} u_{0}\right)$ and

$$
v_{1} \in C^{0}\left(\mathbf{R}, \mathscr{Q}\left(A_{2}\right)\right)
$$

with $A_{2} v_{1}(t)=T_{2}(t) A_{2}\left(u_{1}-A_{1} u_{0}\right)$ and, hence, $v_{1}^{\prime}(t)-A_{2} v_{1}(t)=0 \forall t \in \mathbf{R}$. Finally, the initial conditions $u(0)=u_{0}, u^{\prime}(0)=u_{1}$ can be easily verified from (7) and (8).

Now, it is also easy to check that the same formula (7) gives the solution of $\left(d / d t-A_{1}\right)\left(d / d t-A_{2}\right) u(t)=0, u(0)=u_{0} \in \mathscr{D}_{2}, u^{\prime}(0)=u_{1} \in \mathscr{D}_{1}$, so that the order in which we write the factors in (4) does not matter. ${ }^{1}$

Therefore, if $u(t)$ is a solution of (4), then $v_{1}(t)=u^{\prime}(t)-A_{1} u(t)$ and $v_{2}(t)=u^{\prime}(t)-A_{2} u(t)$ satisfy the first-order equations $v_{1}^{\prime}(t)=A_{2} v_{1}(t)$ and $v_{2}^{\prime}(t)=A_{1} v_{2}(t)$, respectively, and hence,

$$
\left\|v_{1}(t)\right\|=\left\|v_{1}(0)\right\|, \quad\left\|v_{2}(t)\right\|=\left\|v_{2}(0)\right\| \quad \forall t \in \mathbf{R} .
$$

Now, defining the operators $B=\left(A_{1}+A_{2}\right) / 2$ and $D=\left(A_{1}-A_{2}\right) / 2$, we can rewrite (9) as

$$
\begin{aligned}
& \left\|\left(u^{\prime}(t)-B u(t)\right)-D u(t)\right\|^{2}=\left\|\left(u^{\prime}(0)-B u(0)\right)-D u(0)\right\|^{2} \\
& \left\|\left(u^{\prime}(t)-B u(t)\right)+D u(t)\right\|^{2}=\left\|\left(u^{\prime}(0)-B u(0)\right)+D u(0)\right\|^{2}
\end{aligned} \quad \forall t \in \mathbf{R} .
$$

Applying the Parallelogram Law to (10) yields

$$
\begin{aligned}
2\left\{\| u^{\prime}(t)\right. & \left.-B u(t)\left\|^{2}+\right\| D u(t) \|^{2}\right\} \\
& =2\left\{\left\|u^{\prime}(0)-B u(0)\right\|^{2}+\|D u(0)\|^{2}\right\} \quad \forall t \in \mathbf{R} .
\end{aligned}
$$

Therefore, we are naturally led to define "the energy at time $t$ " of a solution of (4) by the formula

$$
E(t)=\left\|u^{\prime}(t)-\frac{A_{1}+A_{2}}{2} u(t)\right\|^{2}+\left\|\frac{A_{1}-A_{2}}{2} u(t)\right\|^{2},
$$

and we have proved

Theorem 1. Assume (5). Then the Cauchy problem for (4) with initial data $u(0)=u_{0} \in \mathscr{D}_{2}, u^{\prime}(0)=u_{1} \in \mathscr{D}_{1}$ has a unique solution given by (7). Furthermore, its total energy (12) is preserved,

$$
E(t)=E(0)=\left\|u_{1}-\frac{A_{1}+A_{2}}{2} u_{0}\right\|^{2}+\left\|\frac{A_{1}-A_{2}}{2} u_{0}\right\|^{2} \quad \forall t \in \mathbf{R} .
$$

1 However, we are not allowed to write $d^{2} u / d t^{2}-\left(A_{1}+A_{2}\right) d u / d t+A_{1} A_{2} u(t)=0$, since according to our definition of solution, it need not be twice differentiable, necessarily. 
REMarks. (1) In the case of the wave equation $\left(d^{2} / d t^{2}+A^{2}\right) u(t)=0$, we have $A_{1}=-A_{2}=i A$ ( $A$ selfadjoint), $T_{2}(t)=T_{1}(-t)$, so that $(5)$ is trivially satisfied and (7) reads

$$
u(t)=\frac{1}{2}\left[T_{1}(t)+T_{1}(-t)\right] u_{0}+\int_{0}^{t} T_{1}(t-2 s) u_{1} d s,
$$

or,

$$
u(t)=\frac{1}{2}\left[T_{1}(t)+T_{1}(-t)\right] u_{0}+\frac{1}{2} \int_{-t}^{t} T_{1}(s) u_{1} d s .
$$

R. Hersh obtained this d'Alembert type formula (among many others for higher-order equations) by a different method in [3]. Also, our definition of "energy" (12) coincides with the usual definition (see [1], [2], [5]) since $\left(A_{1}+A_{2}\right) / 2=0$ and $\left(A_{1}-A_{2}\right) / 2=i A$.

(2) Still in the case of the wave equation, we observe that, if $u_{1}$ is in the range of $A,(6)$ enables us to write (13) as

$$
u(t)=\frac{1}{2}\left[T_{1}(t)+T_{1}(-t)\right] u_{0}+\frac{1}{2}\left[T_{1}(t)-T_{1}(-t)\right](i A)^{-1} u_{1},
$$

or,

$$
u(t)=\frac{1}{2}\left[T_{1}(t)\left(u_{0}+(i A)^{-1} u_{1}\right)+T_{1}(-t)\left(u_{0}-(i A)^{-1} u_{1}\right)\right]
$$

a well-known formula derived by Hille [4]. Of course (as Hersh observes in [3]), (14) has the advantage that it is meaningful even if $u_{1}$ is not in the range of $A$.

(3) Clearly, we do not have to assume (5) in Theorem 1 in order to obtain an existence and uniqueness theorem. That assumption is made to give us conservation of "energy", as defined by (12).

3. Asymptotic equipartition of energy. We start by defining the "Kinetic" and "Potential" energies in (12) by

$$
K(t)=\left\|u^{\prime}(t)-\frac{A_{1}+A_{2}}{2} u(t)\right\|^{2} \text { and } P(t)=\left\|\frac{A_{1}-A_{2}}{2} u(t)\right\|^{2},
$$

respectively. Still assuming $(5)$ and that $u(0)=u_{0} \in \mathscr{D}_{2}, u^{\prime}(0)=u_{1} \in \mathscr{D}_{1}$, we get from (7) that

$$
\begin{aligned}
\left(A_{1}-A_{2}\right) u(t)=\frac{1}{2}\left[T_{1}(t)+T_{2}(t)\right]\left(A_{1}-A_{2}\right) u_{0} \\
\quad+\int_{0}^{t} T_{1}(t-s) T_{2}(s)\left(A_{1}-A_{2}\right)\left(u_{1}-\frac{A_{1}+A_{2}}{2} u_{0}\right) d s \\
=\frac{1}{2}\left[T_{1}(t)+T_{2}(t)\right]\left(A_{1}-A_{2}\right) u_{0}+\left[T_{1}(t)-T_{2}(t)\right]\left(u_{1}-\frac{A_{1}+A_{2}}{2} u_{0}\right) \\
=T_{1}(t)\left(u_{1}-A_{2} u_{0}\right)-T_{2}(t)\left(u_{1}-A_{1} u_{0}\right)
\end{aligned}
$$


the second equality holding in view of (6). Therefore, the "potential energy" is given by

$$
\begin{aligned}
P(t)= & \left\|\frac{A_{1}-A_{2}}{2} u(t)\right\|^{2} \\
= & \frac{1}{4}\left[\left\|u_{1}-A_{2} u_{0}\right\|^{2}+\left\|u_{1}-A_{1} u_{0}\right\|^{2}\right. \\
& \left.\quad-2 \operatorname{Re}\left(T_{1}(t)\left(u_{1}-A_{2} u_{0}\right), T_{2}(t)\left(u_{1}-A_{1} u_{0}\right)\right)\right] .
\end{aligned}
$$

Now, by the same argument used in $\S 2$, we have

$$
\begin{aligned}
\left\|u_{1}-A_{2} u_{0}\right\|^{2}+\left\|u_{1}-A_{1} u_{0}\right\|^{2} & =2\left(\left\|u_{1}-\frac{A_{1}+A_{2}}{2} u_{0}\right\|^{2}+\left\|\frac{A_{1}-A_{2}}{2} u_{0}\right\|^{2}\right) \\
& =2 E(0)
\end{aligned}
$$

and hence,

$$
\begin{aligned}
P(t) & =\frac{1}{4}\left[2 E(0)-2 \operatorname{Re}\left(T_{1}(t)\left(u_{1}-A_{2} u_{0}\right), T_{2}(t)\left(u_{1}-A_{1} u_{0}\right)\right)\right] \\
& =\frac{1}{2} E(0)-\frac{1}{2} \operatorname{Re}\left(T_{1}(t)\left(u_{1}-A_{2} u_{0}\right), T_{2}(t)\left(u_{1}-A_{1} u_{0}\right)\right)
\end{aligned}
$$

This proves

THEOREM 2. If (5) holds and $u(t)$ is the solution of (4) with data $u(0)=u_{0}$ $\in \mathscr{D}_{2}, u^{\prime}(0)=u_{1} \in \mathscr{D}_{1}$, then,

$$
\lim _{|t| \rightarrow \infty} K(t)=\lim _{|t| \rightarrow \infty} P(t)=\frac{1}{2} E(0)
$$

if and only if

$$
\lim _{|t| \rightarrow \infty} \operatorname{Re}\left(T_{1}(t)\left(u_{1}-A_{2} u_{0}\right), T_{2}(t)\left(u_{1}-A_{1} u_{0}\right)\right)=0 .
$$

Now, a few comments on hypothesis (5) are in order. It means that the selfadjoint operators $A_{1} / i$ and $A_{2} / i$ "permute", i.e., that their spectral families commute. Therefore, by a theorem of von Neumann [6, Chapter IX], both $A_{1} / i$ and $A_{2} / i$ are functions of some selfadjoint operator $A$,

$$
A_{1}=i \varphi_{1}(A), \quad A_{2}=i \varphi_{2}(A)
$$

Let $\left\{E_{\lambda}\right\}$ denote the spectral family associated with $A$. Since $T_{1}(t)=\exp \left(t A_{1}\right)$ $=\exp \left(i t \varphi_{1}(A)\right)$ and $T_{2}(t)=\exp \left(t A_{2}\right)=\exp \left(i t \varphi_{2}(A)\right)$, we can write

$$
\begin{aligned}
\left(T_{1}(t) h,\right. & \left.T_{2}(t) h\right)=\int_{-\infty}^{\infty} e^{i t \varphi_{1}(\lambda)} d\left(E_{\lambda} h, T_{2}(t) h\right) \\
& =\int_{-\infty}^{\infty} e^{i t \varphi_{1}(\lambda)} d_{\lambda}\left(\int_{-\infty}^{\infty} \overline{e^{i t \varphi_{2}(\mu)}} d_{\mu}\left(E_{\lambda} h, E_{\mu} h\right)\right) \\
& =\int_{-\infty}^{\infty} e^{i t \varphi_{1}(\lambda)} d_{\lambda}\left(\int_{-\infty}^{\lambda} e^{-i t \varphi_{2}(\mu)} d_{\mu}\left(E_{\mu} h, h\right)\right) \\
& =\int_{-\infty}^{\infty} e^{i t\left[\varphi_{1}(\lambda)-\varphi_{2}(\lambda)\right]} d\left\|E_{\lambda} h\right\|^{2} \quad \forall h \in H .
\end{aligned}
$$


On the other hand, since $\mathscr{D}_{1}$ is dense in $H$, the requirement that the condition

$$
\lim _{|t| \rightarrow \infty} \operatorname{Re}\left(T_{1}(t)\left(u_{1}-A_{2} u_{0}\right), T_{2}(t)\left(u_{1}-A_{1} u_{0}\right)\right)=0
$$

of Theorem 2 hold for all $u_{0} \in \mathscr{D}_{2}, u_{1} \in \mathscr{D}_{1}$ is clearly equivalent to $\lim _{|t| \rightarrow \infty}\left(T_{1}(t) h, T_{2}(t) h\right)=0 \forall h \in H$. Hence, by what we saw above, one has equipartition of energy for any solution $u(t)$ of (4) with $u(0) \in \mathscr{D}_{2}, u^{\prime}(0)$ $\in \mathscr{D}_{1}$ if and only if

$$
\int_{-\infty}^{\infty} e^{i t\left[\varphi_{1}(\lambda)-\varphi_{2}(\lambda)\right]} d\left\|E_{\lambda} h\right\|^{2}=0 \quad \forall h \in H .
$$

We observe that in the special case of the wave equation, we have $A_{1}=-A_{2}=i A$ ( $A$ selfadjoint $)$ so that we choose $\varphi_{1}(\lambda)=\lambda, \varphi_{2}(\lambda)=-\lambda$, and (16) reads

$$
\lim _{|t| \rightarrow \infty} \int_{-\infty}^{\infty} e^{2 i t \lambda} d\left\|E_{\lambda} h\right\|^{2}=0 \quad \forall h \in H
$$

which is the condition presented in [2].

\section{BIBLIOGRAPHY}

1. R. J. Duffin, Equipartition of energy in wave motion, J. Math. Anal. Appl. 32 (1970), 386-391. MR 42 \# 4086.

2. J. A. Goldstein, An asymptotic property of solutions of wave equations, Proc. Amer. Math. Soc. 23 (1969), 359-363. MR 40 \#3365.

3. R. Hersh, Explicit solution of a class of higher-order abstract Cauchy problems, J. Differential Equations 8 (1970), 570-579.

4. E. Hille, Une généralisation du problème de Cauchy, Ann. Inst. Fourier (Grenoble) 4 (1952), 31-48. MR 15, 718.

5. M. Shinbrot, Asymptotic behavior of solutions of abstract wave equations, Proc. Amer. Math. Soc. 19 (1968), 1403-1406. MR 37 \#6801.

6. F. Riesz and B. Sz.-Nagy, Functional analysis, Ungar, New York, 1955. MR 17, 175.

Universidade Federal do Rio de Janeiro, Caixa Postal 1835-ZC-00, 20.000 Rio de JANEIRO, GB, BRASIL

Current address: Universidade de Brasilia, Departamento de Matemática, 70.000 Brasília, DF, Brasil 